\title{
Geodesic knots in cusped hyperbolic 3-manifolds
}

\author{
SALLY M KUHLMANN
}

\begin{abstract}
We consider the existence of simple closed geodesics or "geodesic knots" in finite volume orientable hyperbolic 3-manifolds. Previous results show that at least one geodesic knot always exists [1], and that certain arithmetic manifolds contain infinitely many geodesic knots [2], [5]. In this paper we show that all cusped orientable finite volume hyperbolic 3-manifolds contain infinitely many geodesic knots. Our proof is constructive, and the infinite family of geodesic knots produced approach a limiting infinite simple geodesic in the manifold.
\end{abstract}

57N10, 53C22; 57M50, 30F40

\section{Introduction}

A geodesic in a Riemannian manifold is said to be simple if it has no self-intersections and nonsimple otherwise. In this paper we study geodesics in hyperbolic manifolds, that is, complete Riemannian manifolds of constant curvature -1. Properties of simple closed geodesics in hyperbolic 3-manifolds were first studied by Sakai [6], who introduced the terminology "geodesic knots" to describe them.

A Riemannian manifold may or may not contain simple closed geodesics, and the question of which ones do is open in general. However, the answer is known for hyperbolic manifolds of dimension two and three. In an orientable finite area hyperbolic 2-manifold, each non-contractible non-peripheral simple closed curve is homotopic to a simple closed geodesic, hence the only example not containing a simple closed geodesic is the thrice-punctured sphere. The case of orientable hyperbolic 3-manifolds was solved by Adams, Hass and Scott [1]. They showed that every finite volume orientable hyperbolic 3-manifold contains a geodesic knot, and that the only non-elementary infinite volume exception is the quotient of $\mathbb{M}^{3}$ by a Fuchsian group corresponding to the thrice-punctured sphere.

Since self-intersections of a 1-dimensional loop in a 3-dimensional manifold should be rare, we might expect that most hyperbolic 3-manifolds in fact admit infinitely many geodesic knots. This paper thus addresses the question:

Question 1 Which hyperbolic 3-manifolds contain infinitely many geodesic knots? 
Some partial answers to Question 1 are known. Chinburg and Reid [2] showed that there exist infinitely many noncommensurable closed hyperbolic 3-manifolds all of whose closed geodesics are simple, thus providing examples of arithmetic hyperbolic 3manifolds containing infinitely many geodesic knots. In [5] we showed using arithmetic methods that if $M=\mathbb{H}^{3} / \Gamma$ is a hyperbolic 3-orbifold such that $\Gamma$ is a finite index subgroup of the Bianchi group $\Gamma_{d}=\mathrm{PSL}_{2}\left(\mathcal{O}_{d}\right)$ for some square-free positive integer $d$, then $M$ contains infinitely many geodesic knots. In this paper we take a geometric approach and prove a general result for cusped hyperbolic 3-manifolds, that is, noncompact hyperbolic 3-manifolds of finite volume.

Theorem 1.1 Every cusped orientable hyperbolic 3-manifold contains infinitely many geodesic knots.

The idea is to consider infinite families of closed geodesics which approach a limiting infinite simple geodesic in the manifold, and to show that infinitely many of these closed geodesics are embedded. This idea can be illustrated one dimension down. Consider an infinite cusp-to-cusp geodesic in a cusped hyperbolic 2-manifold. This geodesic can be perturbed slightly to form a closed geodesic which spirals some distance towards the cusp before spiralling back and closing up, as in Figure 1. By varying the amount of spiralling, we can obtain infinitely many such closed geodesics. Moreover, with the extra dimension in three-dimensional cusped manifolds, these geodesics should also typically avoid self-intersection.

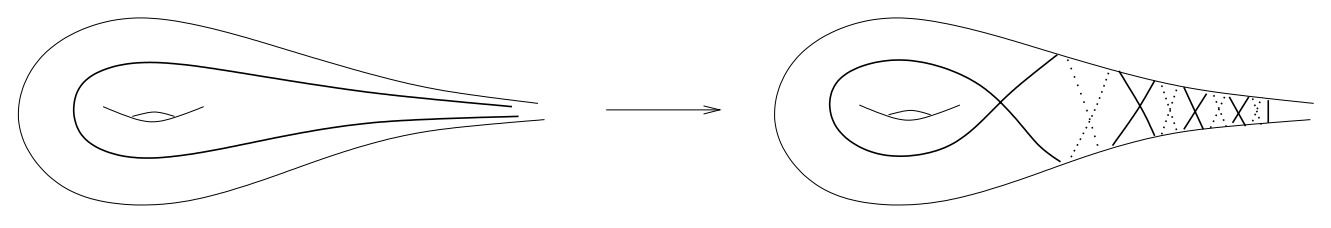

Figure 1: A cusp-to-cusp geodesic can be perturbed slightly to form a closed curve, spiralling some way out into the cusp.

The work in this paper was part of the $\mathrm{PhD}$ thesis [4], where an existence result for geodesic knots in closed hyperbolic 3-manifolds is also proved. In particular it is shown that if a closed hyperbolic 3-manifold satisfies certain geometric and arithmetic conditions, then it contains infinitely many geodesic knots. The conditions on the manifold are checkable, and have been verified for many manifolds in the HodgsonWeeks closed census. This result will appear in the subsequent paper [3].

This was partially supported by an Australian Postgraduate Award. I wish to thank Craig Hodgson for his suggestions and help. 


\section{An infinite family of closed geodesics}

To prove Theorem 1.1, we start by defining a suitable class of spiralling closed geodesics in dimension three. Let our cusped hyperbolic 3-manifold be $M=\mathbb{H}^{3} / \Gamma$ for a discrete torsion-free group of isometries $\Gamma \subset \operatorname{Isom}^{+}\left(\mathbb{M}^{3}\right)$. Choose a cusp $C$ of $M$ and expand a horoball neighbourhood of it with torus boundary. The maximal embedded horoball neighbourhood $U$ has boundary $T$ a torus with a finite number of self-tangencies Pick a point of self-tangency, a bumping point $A$; then let $\gamma_{\infty}$ be the infinite geodesic passing from $C$ to itself through $A$, perpendicular to $T$, as shown schematically in Figure 2.

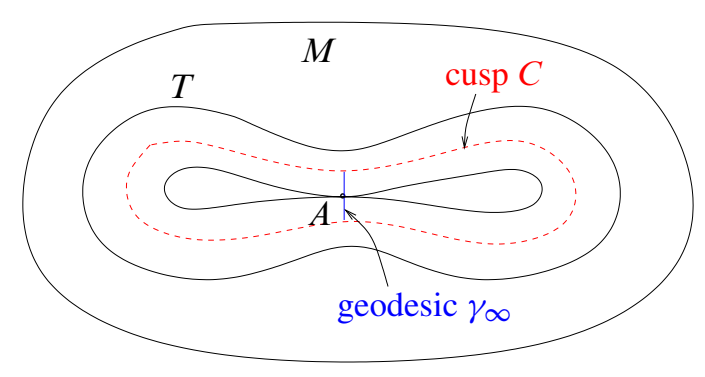

Figure 2: The infinite cusp-to-cusp geodesic $\gamma_{\infty}$

Consider a lift $\widetilde{A} \in \mathbb{H}^{3}$ of the bumping point $A$ under the covering projection $\pi: \mathbb{H}^{3}=$ $\widetilde{M} \rightarrow M$. Then the preimage $\pi^{-1}(T)$ of $T$ contains two horospheres say $H$ and $H^{\prime}$ which intersect precisely at $\widetilde{A}$. See Figure 3 . Since they both cover $T, H$ and $H^{\prime}$ are

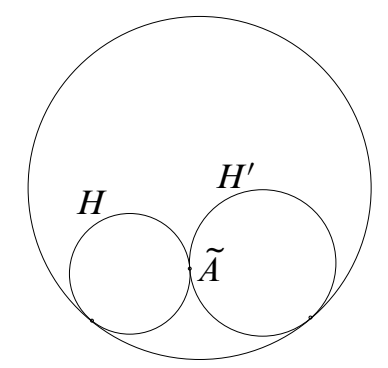

Figure 3: Adjacent horospheres $H$ and $H^{\prime}$ in the Poincaré ball model of $\mathbb{M}^{3}$

related by certain isometries in $\Gamma$. The closed geodesics that we consider in $M$ are those corresponding to all possible isometries in $\Gamma$ mapping $H$ to $H^{\prime}$.

Algebraic $8 \mathcal{G}$ Geometric Topology, Volume 6 (2006) 
Let $g \in \Gamma$ be an arbitrary such isometry taking $H$ to $H^{\prime}$, and choose generators $a, b \in \Gamma$ for the cusp group of $C$ which fix $H^{\prime}$ in $\mathbb{H}^{3}$. Then the isometries in $\Gamma$ mapping $H$ to $H^{\prime}$ are precisely those composed of

- an isometry fixing $H$, followed by

- any given isometry mapping $H$ to $H^{\prime}$, followed by

- an isometry fixing $H^{\prime}$.

This yields an element of the form

$$
\begin{aligned}
a^{p_{1}} b^{q_{1}} \cdot g \cdot\left(g^{-1} a g\right)^{p_{2}}\left(g^{-1} b g\right)^{q_{2}} & =a^{p_{1}} b^{q_{1}} a^{p_{2}} b^{q_{2}} g & & \text { for } p_{i}, q_{i} \in \mathbb{Z} \\
& =a^{p} b^{q} g & & \text { for } p, q \in \mathbb{Z} \\
& =: g_{p, q} & &
\end{aligned}
$$

Denote the geodesic axis in $\mathbb{H}^{3}$ corresponding to $g_{p, q}$ by $\widetilde{\gamma}_{p, q}$, and the closed geodesic in $M$ by $\gamma_{p, q}$. So for any given $g, a$ and $b$, the set of geodesics corresponding to all possible isometries mapping $H$ to $H^{\prime}$ can be parametrised by $\mathbb{Z} \times \mathbb{Z}$. Later we will make a canonical choice for $g$, given elements $a$ and $b$ generating the cusp group, and will see that as $|(p, q)| \rightarrow \infty$, the geodesic axes $\widetilde{\gamma}_{p, q}$ approach a lift of the infinite geodesic $\gamma_{\infty}$. This limiting behaviour is in fact independent of the choice of $g, a$ and $b$.

\section{A normalised preimage in $\mathbb{H}^{3}$}

To study the limiting behaviour of the geodesics $\gamma_{p, q}$ and hence show that infinitely many are simple, we normalise the position of the preimage of $T$ in $\mathbb{H}^{3}$. To do this we must first make more precise our notation for the generators $a, b$ of the cusp group, and their corresponding loops in $M$.

Let $\widehat{T}_{r}$ denote the embedded horospherical torus around $C$ which is hyperbolic distance $r$ further into the cusp than $T$. Then $a$ and $b$ correspond to elements of $\pi_{1}\left(\widehat{T}_{r}\right)$. Pick an $r>0$. Then there is a projection map $\hat{\pi}: \widehat{T}_{r} \rightarrow T$ mapping points radially from the cusp, so that two points $\widehat{A}$ and $\widehat{B}$ on the nonsingular torus $\widehat{T}_{r}$ are mapped to the singular point $A$ of $T$, as shown in Figure 4. Let $\widehat{\alpha}, \widehat{\beta} \in \pi_{1}\left(\widehat{T}_{r}\right)$ be loops on $\widehat{T}_{r}$ representing $a$ and $b$ respectively. Since $a$ and $b$ generate the cusp group, $\widehat{\alpha}$ and $\widehat{\beta}$ are dual oriented non-contractible simple closed curves on $\widehat{T}_{r}$ which after isotopy we may assume are Euclidean geodesics with common basepoint $\widehat{A}$. We will generally identify these curves with their image curves $\alpha:=\hat{\pi}(\widehat{\alpha})$ and $\beta:=\hat{\pi}(\widehat{\beta})$ on $T$, pulling back to $\widehat{T}_{r}$ to avoid complications arising from the self-tangencies of $T$. 


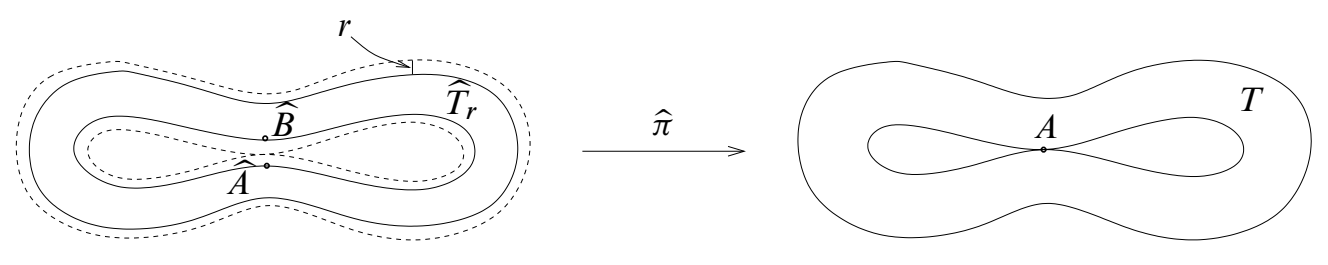

Figure 4: The projection map $\widehat{\pi}: \widehat{T}_{r} \rightarrow T$ maps two points $\widehat{A}$ and $\widehat{B}$ to $A$.

We now work in the upper half-space model of $\mathbb{H}^{3}$, and regard $\Gamma \cong \pi_{1}(M)$ as a subgroup of $\mathrm{PSL}_{2}(\mathbb{C})$. Let $\mathbb{C}_{\infty}=\mathbb{C} \cup\{\infty\}$ denote the sphere at infinity of the upper half-space model of $\mathbb{M}^{3}$, and let the horosphere centred at $z \in \mathbb{C}_{\infty}$ be $H_{z}$. The preimage $\pi^{-1}(T)$ of $T$ is a union of horospheres meeting tangentially at the preimage of the singular point(s) of $T$. By an isometry, normalise so that $H^{\prime}$ is the horizontal horosphere $H_{\infty}$ at height 1 , and $H$ is the horosphere $H_{0}$, tangent to $H^{\prime}$. Parametrise $H_{\infty}$ by $\mathbb{C}$ to match with $\mathbb{C}_{\infty}$ under vertical translation.

The preimages of the curves $\alpha, \beta \subset T$ determine a tiling of each horosphere $H_{z} \subset$ $\pi^{-1}(T)$ by parallelograms. Each parallelogram contains two points covering the bumping point $A \in M$, corresponding to $\widehat{A}$ (at the vertex of the parallelogram) and $\widehat{B}$ in $\widehat{T}_{r}$. On $H_{\infty}$ we see this as a tiling of the complex plane. Let $t_{\alpha}$ and $t_{\beta}$ be the complex numbers corresponding to translation along lifts of the curves $\alpha$ and $\beta$ on $H_{\infty}$. Thus the parabolic elements $a, b \in \Gamma$ have representations

$$
a=\left(\begin{array}{cc}
1 & t_{\alpha} \\
0 & 1
\end{array}\right) \quad \text { and } \quad b=\left(\begin{array}{cc}
1 & t_{\beta} \\
0 & 1
\end{array}\right)
$$

Let the lift of $A$ at complex coordinate 0 on $H_{\infty}$ be $A_{0,0}$, and let the other lift of $A$ in the parallelogram $\left\{r t_{\alpha}+s t_{\beta}: r, s \in[0,1)\right\} \subset H_{\infty}$ be $B_{0,0}$. For $(p, q) \in \mathbb{Z}^{2}$ let $A_{p, q}$ be the lift of $A$ on $H_{\infty}$ obtained from $A_{0,0}$ by translation by the complex number $a_{p, q}:=p t_{\alpha}+q t_{\beta}$, and define $B_{p, q}$ and $b_{p, q}$ similarly. So the horosphere $H_{a_{p, q}} \subset \pi^{-1}(T)$ is tangent to $H_{\infty}$ at the point $A_{p, q}$ and $H_{b_{p, q}} \subset \pi^{-1}(T)$ is tangent at $B_{p, q}$. See Figure 5 .

It is natural to express points on $H_{\infty}$ by their coordinates with respect to $\alpha$ and $\beta$. Let the point $B_{0,0}$ be given by $b_{0,0}=x_{0} t_{\alpha}+y_{0} t_{\beta}$, where $x_{0}, y_{0} \in[0,1)$ and $x_{0}, y_{0}$ are not both 0 , since $B_{0,0} \neq A_{0,0}$. Then $a_{p, q}=p t_{\alpha}+q t_{\beta}$ and $b_{p, q}=\left(p+x_{0}\right) t_{\alpha}+\left(q+y_{0}\right) t_{\beta}$.

Definition Let $z \in H_{\infty}$ be at complex position $z=x t_{\alpha}+y t_{\beta}$. We call $x$ the $t_{\alpha}$-coordinate of $z$ and $y$ the $t_{\beta}$-coordinate of $z$. 


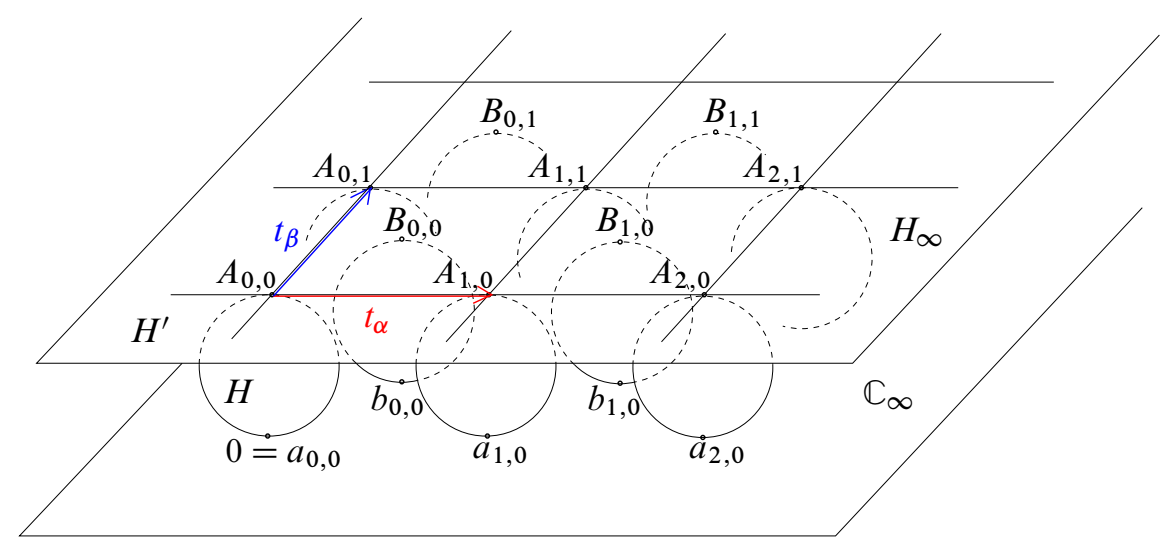

Figure 5: The normalised preimage of $T$ in the upper half-space model of $\mathbb{M}^{3}$

The element $g \in \Gamma$ was chosen as an arbitrary isometry mapping $H=H_{0}$ to $H^{\prime}=H_{\infty}$. Thus $g$ maps

(i) 0 to $\infty$ on the sphere at infinity $\mathbb{C}_{\infty}$, and

(ii) bumping point $A_{0,0}$ to some $B_{p, q}$ on $H_{\infty}$.

Given basis curves $\alpha$ and $\beta$, there is a canonical choice for $B_{p, q}$ in (ii), namely $B_{0,0}$. Then, since the Möbius transformation corresponding to $g$ must map $0=a_{0,0} \mapsto$ $\infty \mapsto b_{0,0}$, we find that $g$ is of the form

$$
g=\left(\begin{array}{cc}
c b_{0,0} & -\frac{1}{c} \\
c & 0
\end{array}\right) \quad \text { for some } c \in \mathbb{C} .
$$

Therefore, with this normalisation the geodesics $\gamma_{p, q}$ are determined by the axes of the isometries

$$
\begin{aligned}
g_{p, q} & =a^{p} b^{q} g \\
& =\left(\begin{array}{cc}
1 & p t_{\alpha}+q t_{\beta} \\
0 & 1
\end{array}\right)\left(\begin{array}{cc}
c b_{0,0} & -\frac{1}{c} \\
c & 0
\end{array}\right) \\
& =\left(\begin{array}{cc}
c b_{p, q} & -\frac{1}{c} \\
c & 0
\end{array}\right)
\end{aligned}
$$

which map horospheres $H_{0}=H_{a_{0,0}} \rightarrow H_{\infty} \rightarrow H_{b_{p, q}}$, taking the point $A_{0,0}$ to $B_{p, q}$. 
Let $\widetilde{\gamma}_{p, q} \subset \pi^{-1}\left(\gamma_{p, q}\right)$ be the axis of this isometry $g_{p, q}$. Then $\widetilde{\gamma}_{p, q}$ has endpoints $z=z_{ \pm} \in \mathbb{C}_{\infty}$ satisfying

$$
\frac{c b_{p, q} z-\frac{1}{c}}{c z}=z
$$

so that

$$
\begin{aligned}
z_{ \pm} & =\frac{c b_{p, q} \pm \sqrt{c^{2} b_{p, q}^{2}-4}}{2 c} \\
& =\frac{b_{p, q}}{2} \pm \sqrt{\left(\frac{b_{p, q}}{2}\right)^{2}-\frac{1}{c^{2}}}
\end{aligned}
$$

Since $\left|b_{p, q}\right|=\left|b_{0,0}+p t_{\alpha}+q t_{\beta}\right| \rightarrow \infty$ as $|(p, q)| \rightarrow \infty$, we observe that

$$
\left|\sqrt{\left(\frac{b_{p, q}}{2}\right)^{2}-\frac{1}{c^{2}}}-\frac{b_{p, q}}{2}\right| \rightarrow 0
$$

as $|(p, q)| \rightarrow \infty$, and hence:

(i) the endpoints $z_{ \pm}$satisfy $z_{-} \rightarrow 0$ and $z_{+}-b_{p, q} \rightarrow 0$, and

(ii) the radius

$$
\left|\sqrt{\left(\frac{b_{p, q}}{2}\right)^{2}-\frac{1}{c^{2}}}\right|
$$

of the Euclidean semicircle giving the geodesic axis $\widetilde{\gamma}_{p, q}$ approaches $\infty$,

as $|(p, q)| \rightarrow \infty$.

By (ii), for large enough $|(p, q)|, \widetilde{\gamma}_{p}, q$ intersects $H_{\infty}$ in two points, say $C_{p, q}$ closer to $z_{-}$and $D_{p, q}$ closer to $z_{+}$, with complex coordinates $c_{p, q}$ and $d_{p, q}$ respectively. Due to (i) and (ii), $c_{p, q} \rightarrow a_{0,0}$ and $d_{p, q}-b_{p, q} \rightarrow 0$, so the points $C_{p, q}$ approach $A_{0,0}$ and the points $g_{p, q}^{-1}\left(D_{p, q}\right)$ approach $g_{p, q}^{-1}\left(B_{p, q}\right)=A_{0,0}$. See Figure 6 .

Therefore,

$$
\text { the } \operatorname{arc}\left(g_{p, q}^{-1}\left(D_{p, q}\right), C_{p, q}\right) \text { approaches the bumping point } A_{0,0},
$$

decreasing in length towards 0 as $|(p, q)| \rightarrow \infty$. So for large enough $|(p, q)|$ this arc does not intersect the preimage $\pi^{-1}(U)$ of the maximal horoball neighbourhood $U$ of the cusp. Henceforth we work only with geodesics $\gamma_{p, q}$ for which $|(p, q)|$ is sufficiently large that this occurs. So projecting to $M, \gamma_{p, q}$ consists of two distinct arcs-the arc $\pi\left(\left[g_{p, q}^{-1}\left(D_{p, q}\right), C_{p, q}\right]\right)$ outside the maximal cusp neighbourhood $U$, and the arc $\pi\left(\left(C_{p, q}, D_{p, q}\right)\right)$ inside the maximal cusp neighbourhood $U$. The arc outside 


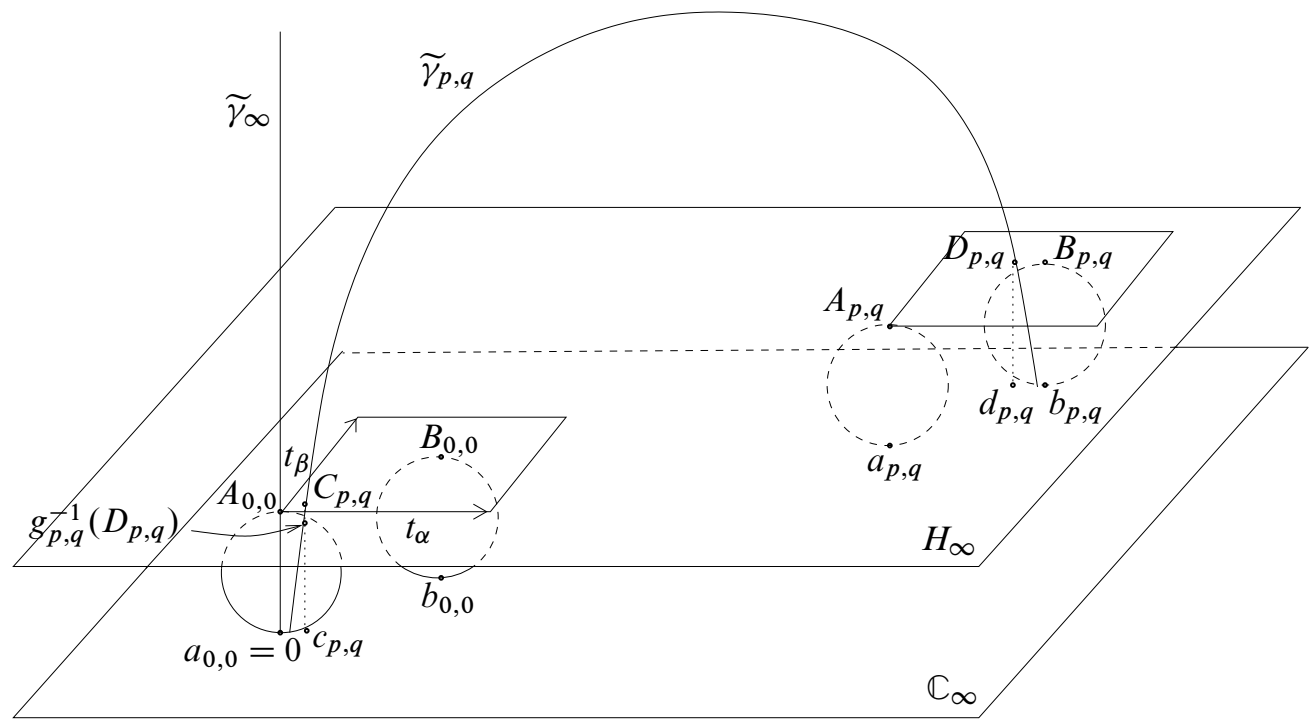

Figure 6: The geodesic axis $\widetilde{\gamma}_{p, q} \subset \pi^{-1}\left(\gamma_{p, q}\right)$ corresponding to the isometry $g_{p, q} \cdot \widetilde{\gamma}_{p, q}$ can be viewed as approaching the vertical axis $\widetilde{\gamma}_{\infty} \subset \pi^{-1}\left(\gamma_{\infty}\right)$ as $|(p, q)| \rightarrow \infty$.

$U$ approaches the bumping point $A$, so we call it the short arc $s_{p, q}$, while the arc inside $U$ increases in length towards infinity as $|(p, q)| \rightarrow \infty$, so is called the long arc $l_{p, q}$. See Figure 7 for a schematic picture.

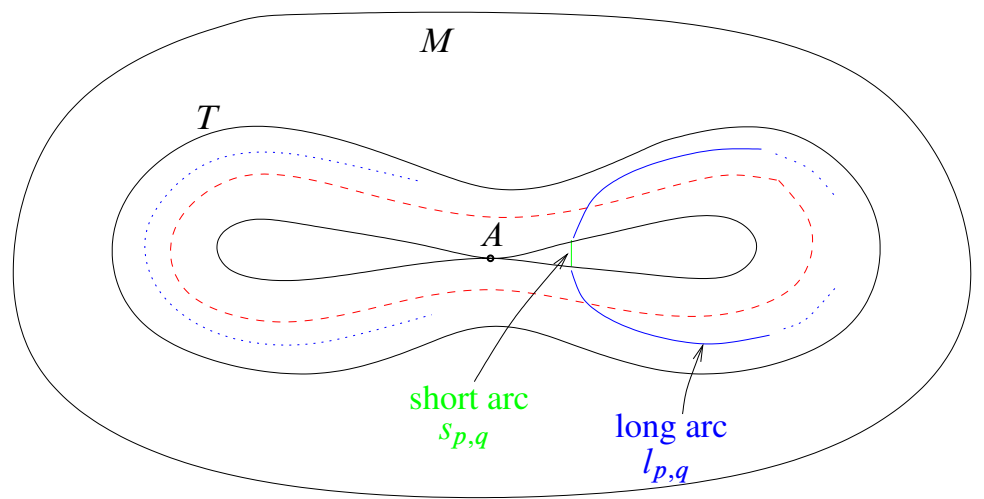

Figure 7: A schematic view of the short and long arcs of a geodesic $\gamma_{p, q}$

Algebraic 83 Geometric Topology, Volume 6 (2006) 
So $\gamma_{p, q}$ is simple precisely when its two arcs $s_{p, q}$ and $l_{p, q}$ are embedded in $M$. We show that this holds for an infinite subfamily of these geodesics.

\section{An infinite subfamily is simple}

Let $\widetilde{s}_{p, q}$ denote the lift $\left[g_{p, q}^{-1}\left(D_{p, q}\right), C_{p, q}\right]$ of the short arc $s_{p, q}$, and $\widetilde{l}_{p, q}$ the lift $\left(C_{p, q}, D_{p, q}\right)$ of the long arc $l_{p, q}$, shown in Figure 6.

Definition Let $R$ be the region in $\mathbb{M}^{3}$ consisting of those points which are closer to the point $A_{0,0}$ than to any other lift of the bumping point $A$. Say that the short arc $s_{p, q}$ is $R$-close to $A$ if its lift $\widetilde{s}_{p, q}$ in $\mathbb{T}^{3}$ is contained in $R$.

By its definition, the projection of $R$ to $M$ is injective, and so $s_{p, q}$ is embedded in $M$ if it is $R$-close to $A$.

Lemma 4.1 In any cusped hyperbolic 3-manifold $M$ all but at most finitely many geodesics $\gamma_{p, q}$ have short arcs which are $R$-close to $A$ and hence embedded.

Proof By its definition, the region $R$ contains a ball $B_{\epsilon}\left(A_{0,0}\right)$ of radius $\epsilon$ around $A_{0,0}$ for some $\epsilon>0$. By (4), $\widetilde{s}_{p, q}$ approaches $A_{0,0}$ as $|(p, q)| \rightarrow \infty$, so there is some $N_{0} \in \mathbb{N}$ such that if $|(p, q)|>N_{0}, \widetilde{s}_{p, q} \subset B_{\epsilon}\left(A_{0,0}\right) \subset R$. Then $s_{p, q}$ is $R$-close to $A$ and hence embedded.

Consider now the long arcs. Isotoping $l_{p, q}$ radially from the cusp to a curve $L_{p, q} \subset T$ corresponds to projecting the lift $\widetilde{l}_{p, q}$ vertically downwards to a line segment, say $\widetilde{L}_{p, q}$ on the horosphere $H_{\infty}$, as in Figure 8.

Lemma 4.2 The long arc $l_{p, q}$ of a geodesic $\gamma_{p, q}$ is nonsimple if and only if the projected line segment $\widetilde{L}_{p, q}$ contains two distinct points which differ by a translation by an integer linear combination of $t_{\alpha}$ and $t_{\beta}$.

Proof Clearly if $l_{p, q}$ has self-intersection the two points on $\widetilde{L}_{p, q}$ giving this selfintersection must project to the same point on $T$, and so differ by an integer linear combination of $t_{\alpha}$ and $t_{\beta}$. Conversely, if any two distinct points on $\widetilde{L}_{p, q}$ differ by an integer linear combination of $t_{\alpha}$ and $t_{\beta}$, then so do two points equidistant from the midpoint of $\widetilde{L}_{p, q}$, by translation along the line segment. Such equidistant points are images of points at the same vertical height on the arc $\widetilde{l}_{p, q}$ and thus the same distance from $T$ on $l_{p, q}$. These points therefore coincide in $M$, producing a self-intersection of $l_{p, q}$. 


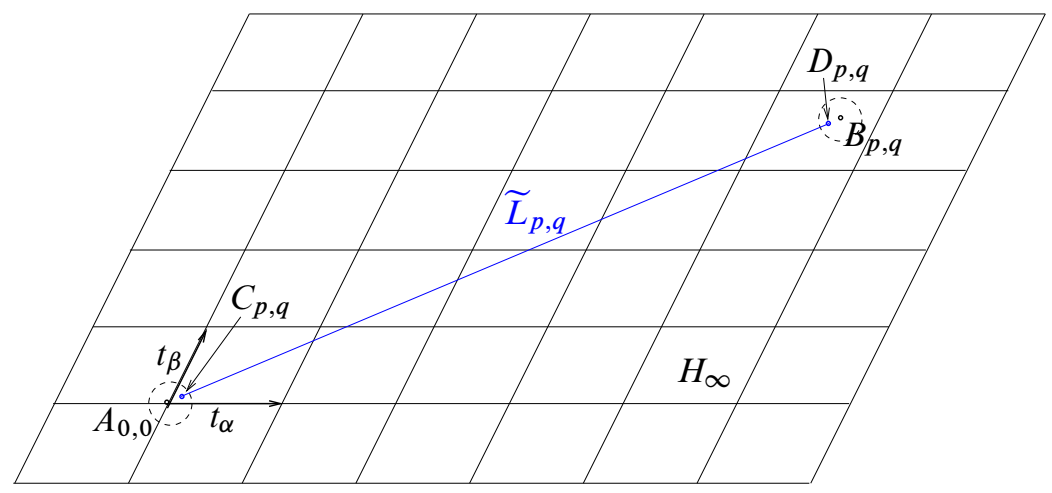

Figure 8: A lift $\widetilde{L}_{p, q}$ of the radial projection of $l_{p, q}$ to $T$

We are now ready to prove Theorem 1.1 which we recall here:

Theorem 1.1 Every cusped orientable hyperbolic 3-manifold contains infinitely many simple closed geodesics.

Proof By Lemma 4.1 it suffices to show that infinitely many geodesics $\gamma_{p, q}$ have embedded long arcs.

Recall that $b_{0,0}=x_{0} t_{\alpha}+y_{0} t_{\beta}$, where $x_{0}, y_{0} \in[0,1)$ and at least one of $x_{0}$ and $y_{0}$ is nonzero. Assume without loss of generality that $y_{0}>0$ - that is, the $t_{\beta}$-coordinate of $b_{0,0}$ is positive.

Consider then the subfamily of geodesics given by $q=0$, ie those given by elements of the form $g_{p, 0}=a^{p} g$. We know that $\left|c_{p, 0}\right|=\operatorname{dist}_{H_{\infty}}\left(A_{0,0}, C_{p, 0}\right)=$ $\operatorname{dist}_{H_{\infty}}\left(B_{p, 0}, D_{p, 0}\right) \rightarrow 0$ as $|p| \rightarrow \infty$. Let $\delta=\min \left\{\frac{1}{2} y_{0}, \frac{1}{2}\left(1-y_{0}\right)\right\}$. Then there exists $P \in \mathbb{N}$ such that for all $p$ with $|p|>P,\left|c_{p, 0}\right|<\delta$. So for $|p|>P$, the complex number $d_{p, 0}-c_{p, 0}$ giving the translation along $\widetilde{L}_{p, 0}$ has $t_{\beta}$-coordinate in the interval $\left(y_{0}-2 \delta, y_{0}+2 \delta\right) \subset(0,1)$. Hence when $|p|>P, \widetilde{L}_{p, 0}$ cannot contain points differing by an integer linear combination of $t_{\alpha}$ and $t_{\beta}$, and so by Lemma 4.2 the long $\operatorname{arc} l_{p, 0}$ is simple and we are done.

Note that the above result cannot be strengthened to show that all but finitely many $\gamma_{p, q}$ are embedded. For example, it is shown in [4] that in the figure-eight knot complement infinite families of nonsimple $\gamma_{p, q}$ occur.

We conclude by remarking that our proof also gives a description of the isotopy classes of the simple geodesics $\gamma_{p, q}$, for $|(p, q)|$ sufficiently large. They are the union of a 
long arc $l_{p, q}$ spiralling inside the maximal cusp neighbourhood $U$, and a short arc $s_{p, q}$ outside this cusp neighbourhood. The long arc can be isotoped to a Euclidean line segment $L_{p, q}$ on $T$, the (singular) torus boundary of $U$, while $s_{p, q}$ is a short connecting arc between the endpoints of $L_{p, q}$, close to the singular point $A$ of $T$. See Figure 9 for a schematic view of this description.

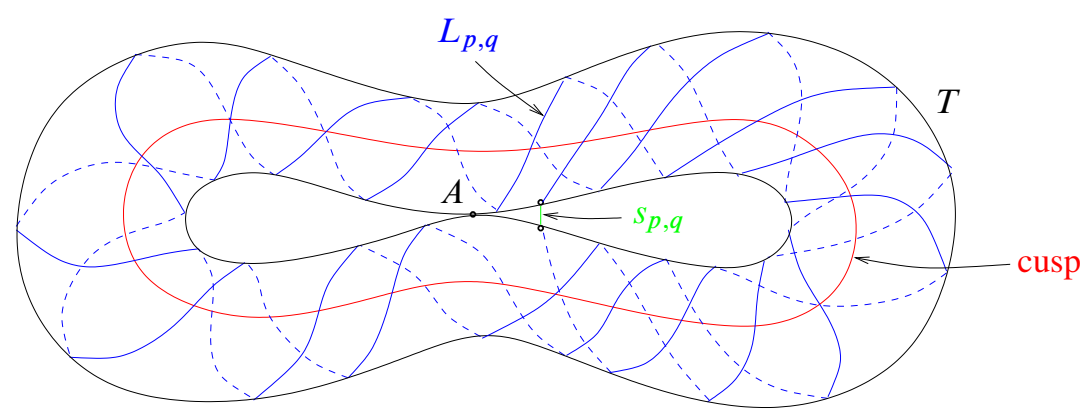

Figure 9: The isotopy class of a geodesic $\gamma_{p, q}$ can be described as the union of a long arc isotoped to lie on the singular torus $T$ and a short connecting arc outside the maximal cusp neighbourhood.

In [4] we investigate this further and obtain precise topological descriptions for the isotopy classes of infinite subfamilies of the geodesics $\gamma_{p, q}$ in any cusped hyperbolic 3-manifold. We also draw explicit projection diagrams for these geodesics in the case of the figure-eight knot complement.

\section{References}

[1] C Adams, J Hass, P Scott, Simple closed geodesics in hyperbolic 3-manifolds, Bull. London Math. Soc. 31 (1999) 81-86 MR1650997

[2] T Chinburg, A W Reid, Closed hyperbolic 3-manifolds whose closed geodesics all are simple, J. Differential Geom. 38 (1993) 545-558 MR1243786

[3] S M Kuhlmann, Geodesic knots in closed hyperbolic 3-manifolds, in preparation

[4] S M Kuhlmann, Geodesic knots in hyperbolic 3-manifolds, $\mathrm{PhD}$ thesis, University of Melbourne (2005) Available at http://eprints.infodiv.unimelb.edu.au/ archive/00001728/

[5] S M Miller, Geodesic knots in the figure-eight knot complement, Experiment. Math. 10 (2001) 419-436

[6] T Sakai, Geodesic knots in a hyperbolic 3-manifold, Kobe J. Math. 8 (1991) 81-87 MR1134707 
Department of Mathematics and Statistics, University of Melbourne Victoria, 3010, Australia

S.Kuhlmann@ms . unimelb.edu . au

Received: 21 April 2006

Algebraic 63 Geometric Topology, Volume 6 (2006) 